\title{
Spreading of a granular droplet under horizontal vibrations
}

\author{
Sidi Mohammed Khefif,,${ }^{1, *}$ Alexandre Valance, ${ }^{2, \dagger}$ and Fouzia Ould-Kaddour ${ }^{3}$ \\ ${ }^{1}$ École Supérieure des Sciences Appliquées, BP 165 RP Bel Horizon, 13000 Tlemcen, Algeria \\ ${ }^{2}$ Institut de physique de Rennes, UMR CNRS 6251, \\ Université de Rennes 1, Campus Beaulieu Bâtiment 11A, \\ 263 av. Général Leclerc, 35042 Rennes Cedex, France \\ ${ }^{3}$ Laboratoire de Physique Théorique \\ Université Abou Bekr Belkaid, BP 11913000 Tlemcen, Algeria
}

(Dated: June 12, 2018)

\begin{abstract}
By means of three-dimensional discrete element simulations, we studied the spreading of a granular droplet on a horizontally vibrated plate. Apart from a short transient with a parabolic shape, the droplet adopts a triangular profile during the spreading. The dynamics of the spreading is governed by two distinct regimes: A super-diffusive regime in the early stages driven by surface flow followed by a second one which is sub-diffusive and governed by bulk flow. The plate bumpiness is found to alter only the spreading rate but plays a minor role on the shape of the granular droplet and on the scaling laws of the spreading. Importantly, we show that in the sub-diffusive regime, the effective friction between the plate and the granular droplet can be interpreted in the framework of the $\mu(I)$-rheology.
\end{abstract}

Keywords: Granular droplet, vibrations, spreading, morphology, rheology

\section{INTRODUCTION}

The spreading dynamics of a granular material subjected to external horizontal agitations may be crucial in some industries like coatings. But unlike the spreading of a liquid on a solid, vibrated granular films have not been given great attention. As a reminder, extensive experimental, theoretical and numerical studies have shown that the spreading dynamics of a liquid droplet goes through two stages, an early capillary slow regime governed by Tanner's law $r \sim t^{1 / 10}$, when the droplet radius $r$ is smaller than the capillary length, followed by a gravitational faster regime $r \sim t^{1 / 7}$, when the droplet has a pancake shape [1]. However, when the viscous dissipation is neglected and a balance between inertia and surface tension is invoked, the power law of the spreading dynamics become $r \sim t^{1 / 2}$ at early stages and $r \sim t^{2 / 3}$ for later ones $[2,3]$. In other studies, it has also been shown that the spreading of polymer nanodroplets in cylindrical geometry scales as $r(t) \sim t^{1 / 7}$ for the earlier times and as $r(t) \sim t^{1 / 5}$ for the asymptotic stages [4-6].

The spreading of a granular system requires in general external mechanical excitation to overcome solid friction. A large number of studies have been conducted on vibrated granular systems but in confined geometries (see for example the review by Nadler et al. [7]). A pioneer work by Sanchez et al. [8] has been conducted to investigate the spreading dynamics of a free granular deposit on a controlled wiggling surface. They show that the transverse width $W$ of the granular film follows a single spreading scaling law $W(t) \sim t^{1 / 3}$ and that the droplet morphology obeys a scale invariant parabolic pro-

\footnotetext{
*sm.khefif@essa-tlemcen.dz

† alexandre.valance@ipr.univ-rennes.fr
}

file. They also proposed a nonlinear diffusion model for the spreading dynamics that reproduces reasonably well the experimental outcomes. From a simple Coulomb frictional model, they were able to identify two different rheological behaviors of the granular droplet: shear thickening at low vibration energies and shear thinning at high energies, leading to a non-monotonous behavior of the effective friction coefficient $\mu$ as a function of the inertial number $I$ of the $\mu(I)$-rheology, in contrast with the previously observed shear-rate dependent behavior in granular inertial regime [9].

One major limitation to this specific experiment is that the spreading process and the droplet rheology are inferred only from the temporal evolution of the droplet shape but not from bulk properties. In order to overcome this limitation and get a better understanding of the underlying physical mechanisms, we perform extensive discrete element method (DEM) simulations of the spreading of a granular droplet on a horizontally vibrated plate. We investigate the spreading dynamics as well as the friction between the droplet and the basal substrate for a wide range of vibration energies and various plate bumpiness. We also analyze the bulk properties of the granular droplet during the spreading process, including velocity field and solid packing fraction.

The paper is organized as follows. In section II we briefly recall the basic elements of the DEM and describe the physical simulated system. Results are presented in section III which includes the morphodynamics of the granular droplet, the scaling laws of the spreading, the influence of the plate bumpiness on the spreading process and the effective rheology of the granular droplet. Conclusion and outlook are given in section IV. 


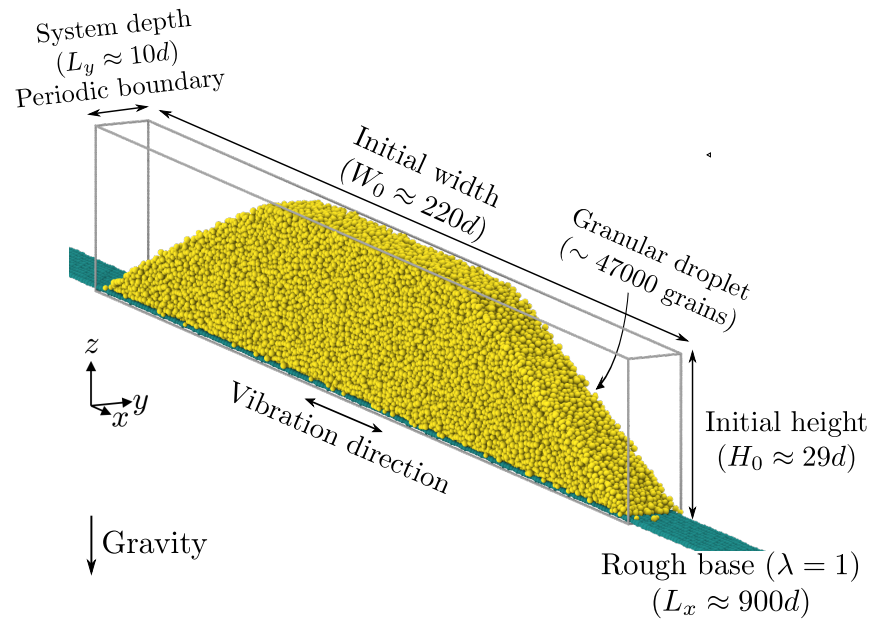

FIG. 1. Sketch of the DEM simulated granular droplet laid on a rough base driven by horizontal vibrations and mimicking the experiment in [8].

\section{SIMULATION PROCEDURE}

\section{A. Simulated system}

We perform 3D discrete element method (DEM) simulations on model systems of polydisperse $( \pm 20 \%)$, cohesionless spheres of diameters uniformly distributed around the mean value $d$ and of uniform material density $\rho$. The simulation box is rectangular $(900 d \times 10 d \times 30 d)$ with periodic boundary conditions along the $y$ dimension; it has free boundaries in the $x$ dimension and is limited in the vertical $z$ dimension by a base and an open top as it is depicted in Fig. 1. The base is made either bumpy or smooth. We employ four different bases: three that are bumpy and a smooth one. The bumpy bases are made of spherical grains placed at the nodes of a square lattice. The average diameter of the basal spheres is $d_{s}=\lambda d$, with $\lambda=0.25,0.5$ and 1 . The smooth base corresponds to $\lambda=0$. The substrate is vibrated horizontally by imposing a sinusoidal movement $x(t)=a \sin (\omega t)$, where $a$ and $\omega$ are respectively the amplitude and the pulsation of the vibration. On the basis of the experiments from Sanchez et al. [8], we set $a=d$ and investigate a set of pulsations ranging from $0.5 \sqrt{g / d}$ to $2.5 \sqrt{g / d}$; where $g$ is the gravity acceleration. In terms of the reduced acceleration $\Gamma=a \omega^{2} / g$, we thus probe values from $0.4 \mathrm{~g}$ to $6 \mathrm{~g}$ for the latter. We undertook a few simulations with thicker systems $(20 d)$. We did not notice any major variations in the spreading dynamics in comparison with small systems $(10 d)$. We thus assume that $10 d$ is large enough to avoid the effects of the periodic boundary conditions.

In the presence of a gravitational field $\boldsymbol{g}$, the grains have translational and rotational accelerations determined by numerically integrating Newton's second law using Velocity Verlet algorithm [10]. The total forces and torques acting on a particle $i$ are:

$$
\begin{gathered}
\boldsymbol{F}_{i}^{\text {total }}=m_{i} \boldsymbol{g}+\sum_{j} \boldsymbol{F}_{i j}^{n}+\boldsymbol{F}_{i j}^{t}, \\
\boldsymbol{\tau}_{i}^{\text {total }}=-\frac{1}{2} \sum_{j} \boldsymbol{r}_{i j}^{n} \times \boldsymbol{F}_{i j}^{t},
\end{gathered}
$$

where index $j$ goes over all the grains in mechanical contact with the grain $i$ and the superscripts $n$ and $t$ stand for normal and tangential components of the contact force. The forces $\boldsymbol{F}^{n}$ and $\boldsymbol{F}^{t}$ are functions of the relative position of the particles and also of their relative velocity, they are composed of conservative (elastic) and dissipative (viscous) parts which can be referred to as a spring-dashpot model. The normal and tangential contact forces are written as [11]:

$$
\begin{gathered}
\boldsymbol{F}^{n}=\left(k_{n} \delta+\gamma_{n} v_{n}\right) \boldsymbol{u}_{n}, \\
\boldsymbol{F}^{t}=\left(k_{t} \xi+\gamma_{t} v_{t}\right) \boldsymbol{u}_{t},
\end{gathered}
$$

where $\delta$ is the overlap distance, $k_{n, t}$ and $\gamma_{n, t}$ are the elastic and the viscoelastic constants and are defined below. The unit vector of the normal direction is $\boldsymbol{u}_{n}=$ $\left(\boldsymbol{r}_{i}-\boldsymbol{r}_{j}\right) /\left|\boldsymbol{r}_{i}-\boldsymbol{r}_{j}\right|$ and $\boldsymbol{u}_{t}$ is its tangential counterpart. $v_{n}$ and $v_{t}$ are, respectively, the moduli of the normal and the tangential components of the relative velocity at contact:

$$
\begin{gathered}
\boldsymbol{v}_{n}=\left(\boldsymbol{v}_{i}-\boldsymbol{v}_{j}\right) \cdot \boldsymbol{u}_{n} \boldsymbol{u}_{n}, \\
\boldsymbol{v}_{t}=\boldsymbol{v}_{i}-\boldsymbol{v}_{j}-\boldsymbol{v}_{n}+\frac{1}{2}\left(d_{i} \boldsymbol{\omega}_{i}+d_{j} \boldsymbol{\omega}_{j}\right) \times \boldsymbol{u}_{n},
\end{gathered}
$$

where the parameters $d_{i}, \boldsymbol{v}_{i}$ and $\boldsymbol{\omega}_{i}$ represent, respectively, the diameter, velocity and angular velocity of the particle $i$. We can thus define a tangential unit vector by $\boldsymbol{u}_{t} \equiv \boldsymbol{v}_{t} / v_{t}$. We denote by $\xi$ the relative elastic tangential displacement between two particles in contact, it is computed by integrating the tangential relative velocity during the lifetime of a contact that started at time $t_{0}: \xi=\int_{t_{0}} v_{t}\left(t^{\prime}\right) d t^{\prime}$. The tangential elongation $\xi$ has to be truncated whenever necessary to satisfy the Coulomb's friction law locally $F_{t} \leq \mu_{p} F_{n}$, where $\mu_{p}$ is the inter-particle friction coefficient and $F_{n} \equiv\left\|\boldsymbol{F}_{n}\right\|$ and $F_{t} \equiv\left\|\boldsymbol{F}_{t}\right\|$. This force scheme is identically applied to the particle-substrate interactions but with a different set of model parameters. In Table I, we give a set of interaction parameters used in the simulations.

Solid mechanics provides relations between the model parameters. When two grains enter in collision, the linear spring-dashpot model of the forces keep the two grains in contact for a finite duration before they separate again. The ratio of the relative normal velocities before and after the collision defines a normal restitution coefficient $e_{n}$ which is directly related to $\gamma_{n}$ by [11]:

$$
e_{n}=\exp \left(-\frac{\gamma_{n}}{2 m_{\mathrm{eff}}} t_{n}\right)
$$


with

$$
t_{n}=\pi\left(\frac{k_{n}}{m_{\mathrm{eff}}}-\left(\frac{\gamma_{n}}{2 m_{\mathrm{eff}}}\right)^{2}\right)^{-1 / 2}
$$

the duration of the collision and $m_{\mathrm{eff}}=m_{i} m_{j} /\left(m_{i}+m_{j}\right)$ the effective mass. Similarly, we can define a restitution coefficient $e_{t}$ for the tangential relative velocities. The restitution coefficients $e_{n}$ and $e_{t}$ set the values of the model parameters $\gamma_{n}$ and $\gamma_{t}$. We choose for $e_{n}$ and $e_{t}$ values corresponding to spent glass beads [12] as reported in Table I.

The value of the spring stiffness should in principle be related to material properties. A link to the Young modulus and Poisson ratio is possible for Hertzian contacts. For linear models, we have to rely on ad hoc approximation [13]. In the linear spring-dashpot model, taking equal normal and tangential contact durations leads to a relation between the elastic constants $k_{n}$ and $k_{t}$ that reads: $7 k_{t}\left(\pi^{2}+\left(\ln e_{n}\right)^{2}\right)=2 k_{n}\left(\pi^{2}+\left(\ln e_{t}\right)^{2}\right)$. This is different from the usual $k_{t} / k_{n}=2 / 7$ for equal restitution coefficients $e_{n}$ and $e_{t}$. We employ a classical value used in the literature $[13,14]: k_{n}=2 \times 10^{5} \mathrm{mg} / \mathrm{d}$ (see Table I). This value corresponds to softer particles than real glass particles but allow faster simulations. We pick the particle-particle and particle-wall friction coefficients to be both $\mu_{p}=0.5$, which corresponds to standard values found in the literature based on static rather than dynamic values [15-17]. Finally, it is noteworthy to say that all the simulation results are given in dimensionless units obtained by setting the particle diameter $d$, its mass $m$ and gravity acceleration $g$ equal to unity.

\section{B. Preparation of the droplet}

We conduct numerical simulations to mimic the spreading experiments of a granular droplet reported in [8]. In order to build up the initial configuration, we add inside the simulation box two transverse walls at symmetric $y$ positions from the middle of the simulation box and are placed 150d apart. We then fill the inner box by pouring 47000 grains from a randomly diluted simple cubic lattice. Once the grains have sedimented, we vigorously shake the whole simulation box horizon-

\begin{tabular}{|c|c|c|c|c|}
\hline Parameter & symbol & grain/grain & grain/base & units \\
\hline Elastic constant & $\overline{k_{n}}$ & $2 \times 10^{5}$ & $2 \times 10^{5}$ & $\overline{\overline{m g / d}}$ \\
\hline Normal restitution & $e_{n}$ & 0.972 & 0.8 & \\
\hline Tangential restitution & $e_{t}$ & 0.25 & 0.35 & \\
\hline Particle friction & $\mu_{p}$ & 0.5 & 0.5 & \\
\hline
\end{tabular}

TABLE I. Interaction values used in the simulations. The particle mass $m$, its diameter $d$ and the gravitation constant $g$ are used to rescale all the material parameters and hence are set to unity. A polydispersity in the particle size of $\pm 20 \%$ is introduced to hinder crystallization. tally with a dimensionless acceleration $\Gamma=4.38$ during a time span $t=130 T$ where $T=2 \pi / \omega$ is the vibration period. After turning off the vibrations and letting the system relax, we slowly move apart the transverse lateral walls until they reach the limits of the simulation box. At the end of the process, the transverse walls are no more in contact with the granular droplet so that they can be removed safely. By doing so, we obtain a granular pile with two slip faces and a flat top which is $220 \times d$ wide, $29 \times d$ high and $10 \times d$ deep (see Fig. 1). In the experiment led in [8], the top surface of the initial pile preparation has the shape of a chalet roof. This is due to a different preparation scheme in the experiment in which a bottomless box is first filled with grains, then horizontally vibrated to level out the deposit before it is lifted up. Our procedure is employed for generating initial configurations of all simulations. The generated granular piles may slightly differ according to the basal bumpiness. For every basal bumpiness corresponding to different values of the parameter $\lambda$, we run the same set of simulations for the following set of dimensionless accelerations $\Gamma=\{0.39,0.49,0.62,0.80,1.10,1.58,2.47,4.39,6.32\}$.

\section{RESULTS}

We present first the simulation results in the case where the basal bumpiness parameter is $\lambda=1$ and then investigate the influence of the bumpiness on the spreading dynamics. Finally, we analyze the rheological property of the granular droplet in course of the spreading process.

\section{A. Droplet morphology}

As already described in the previous section, the initial state is a granular heap with two slip faces and a nearly flat top. When turning on the vibrations, and after a short transient regime of about $t=3 T$, the free surface of the droplet rounds out. The granular droplet profile is shown in Fig. 2 at different times while spreading over a substrate driven by horizontal vibrations. After this transient, we record the droplet profile at regularly spaced time intervals measured in terms of multiples of of the vibration period $T$. The vibrations are turned off when the toe of the granular droplet reaches the limits of the simulation box.

Due to the invariance in the $y$ direction, we focus our attention on the morphological properties of the onedimensional profile $z(x)$ of the granular droplet. From the profile, it is easy to extract the maximum height $H$ and lateral width $W$, and study their temporal evolution in course of time.

In Fig. 3, we present the time evolution of the onedimensional profile of the free surface obtained at the acceleration $\Gamma=0.39$. The solid lines correspond to the best parabolic fits of the numerical data. At the very be- 


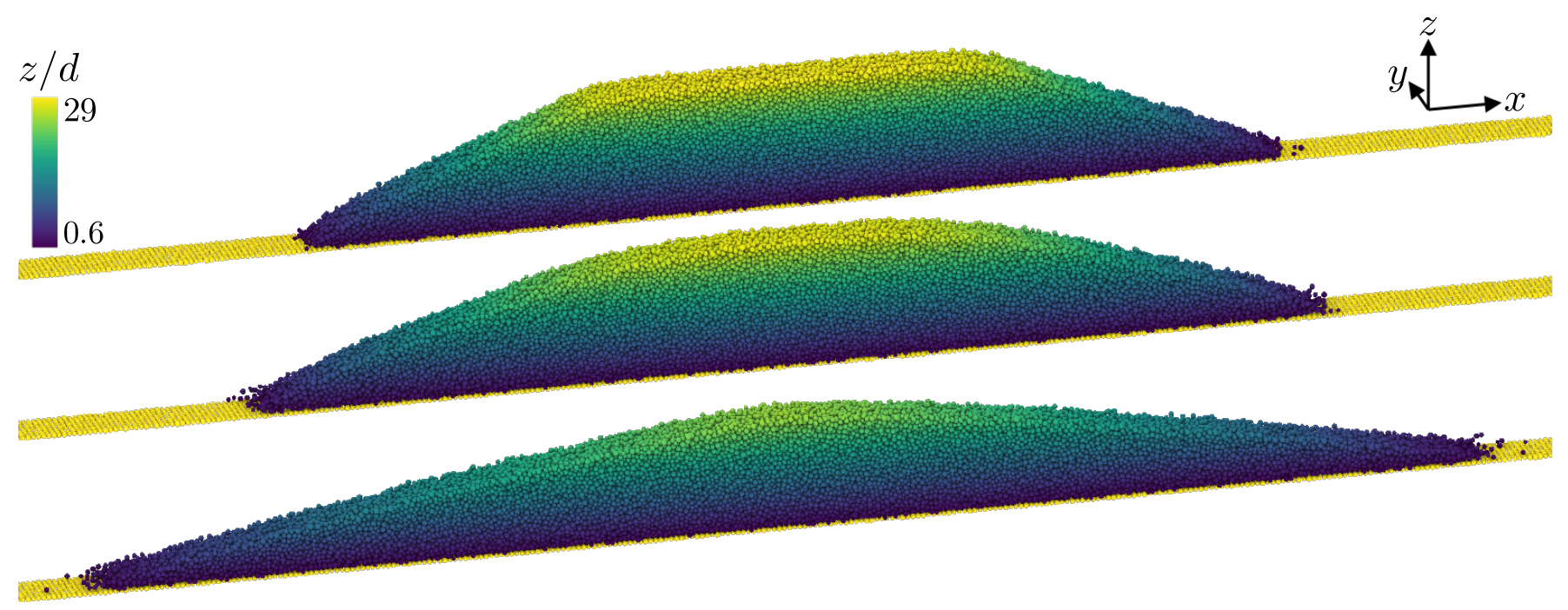

FIG. 2. Profiles of a granular droplet while spreading at acceleration $\Gamma=1.10$ on a substrate of bumpiness $\lambda=1$. The chronological sequences have been recorded at times represented in units of the vibration period: $t / T=1 / 8($ top), $t / T=3$ (middle) and $t / T=12$ (bottom). The color gradient codes the $z$-position of the grains in the droplet. These simulation snapshots have been rendered by the open visualization tool OviTo [18].

ginning of the spreading process, while still in the transient regime $(t / T=1 / 8)$, we clearly see that the heap has a nearly flat top which is progressively smoothed out. The heap then adopts a shape that can be pretty well approximated by a parabolic profile at time $t / T=3$.

At later times, the droplet profile deviates significantly from the parabolic shape and exhibits a triangular morphology. This is the first notable discrepancy with the experiments in [8] where the heap keeps a parabolic shape during the entire spreading process. It is noteworthy to mention that the scale of the vertical dimension in Fig. 3 have been magnified by a factor ten in comparison with the horizontal scale, so that the deviations from the parabolic plots are visually amplified. The profiles

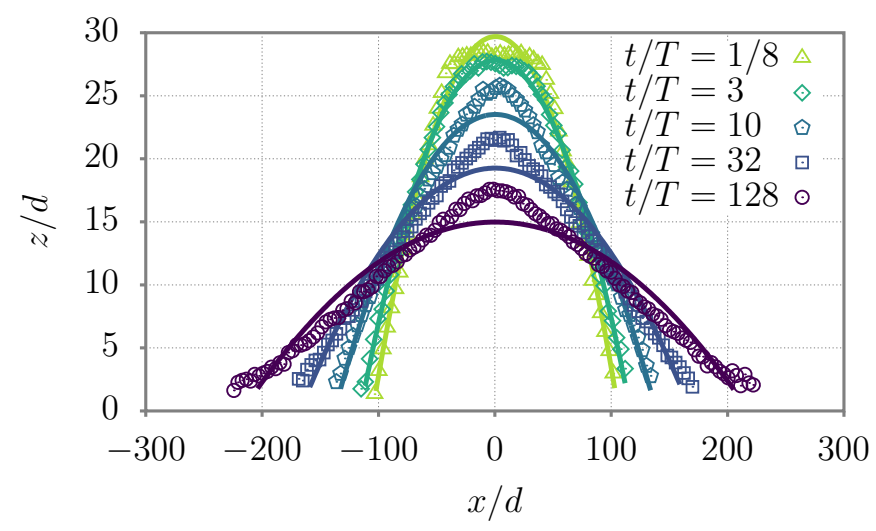

FIG. 3. Mean two-dimensional profiles (open symbols) of a granular droplet at different instants along with their corresponding best parabolic fits (solid lines) for $\Gamma=0.39$ and substrate bumpiness $\lambda=1$. Note the large discrepancy between the horizontal and the vertical scales. rescaled by their respective maximum height $H(t)$ and width $W(t)$ are shown in Fig. 4 . In the rescaled plot, we clearly see the transition from a parabolic shape at the initial stages to a triangular one at the final stages.

\section{B. Spreading dynamics}

A key feature to understand the spreading dynamics of a granular droplet is to quantify the rate of the spreading process as a function of the intensity of the vibrations. In Fig. 5, we present the time evolution of the height $H(t)$ and width $W(t)$ of the granular droplet versus the reduced acceleration $\Gamma$. As expected, the height is de-

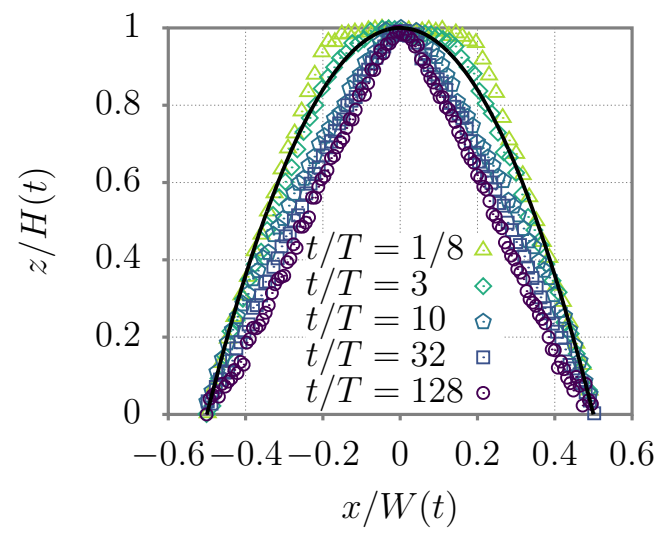

FIG. 4. The granular droplet profiles of Fig. 3 are normalized here by their instantaneous maximum width $W(t)$ and height $H(t)$ (open symbols). The black solid line represents a normalized parabolic function $z(x)=1-(2 x)^{2}$ added to the plot as a guide to the eye. 

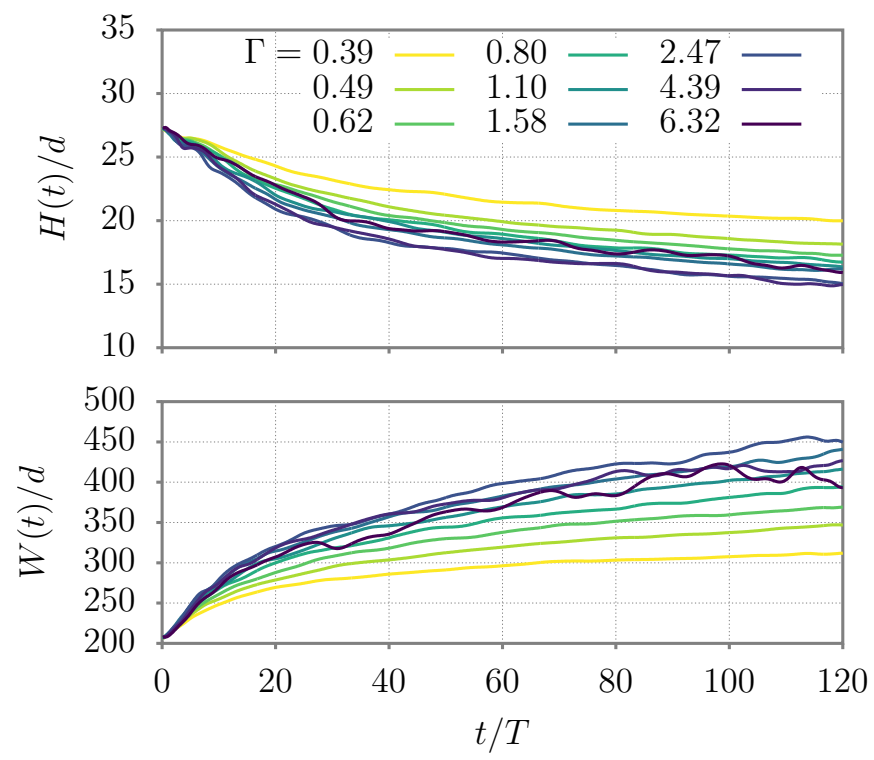

FIG. 5. Time evolution of a granular droplet height $H(t)$ and its width $W(t)$ while horizontally vibrating a rough substrate $(\lambda=1)$ at different acceleration rates $\Gamma$.

creasing in course of time while the width is increasing. We also find that the spreading rate increases with an increasing acceleration $\Gamma$. The strong correlation between the height and width of the granular droplet indicates that its volume keeps roughly constant. In other words, compaction or decompaction process remain marginal. We will come back on that issue later in the paper.

It is noteworthy to mention that in the first stages of the spreading process (i.e., $t \lesssim 5 T$ ), the curves $H(t)$ and $W(t)$ obtained for different values of $\Gamma$ all collapse in a unique trend. This transient regime thus seems to be independent of the amount of vibrational energy injected in the system. These earlier stages correspond in fact to a slight decompaction process as discussed later. After this transient, the higher the acceleration rate is, the more

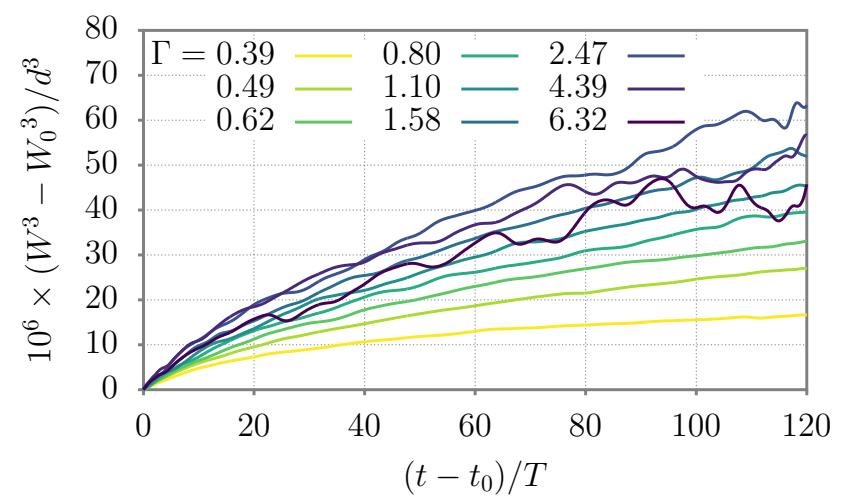

FIG. 6. Spreading dynamics of a granular droplet on a rough substrate $(\lambda=1)$ following the model proposed in [8] (Fig. 3d therein) at different acceleration rates $\Gamma$. the spreading is effective, except for extreme acceleration values $(\Gamma=4.39$ and 6.32$)$ for which the spreading rate saturates or even slows down.

The experiments of Sanchez et al. [8] reveal that the spreading dynamics is sub-diffusive and obeys the following scaling law:

$$
W^{3}(t)-W^{3}(0) \sim t
$$

To check whether this scaling law holds or not, we replot our data to represent the temporal evolution of the relative width $W^{3}(t)-W^{3}\left(t_{0}\right)$ as represented in Fig. 6 . We discard the initial transient and set the initial time to $t_{0}=5 T$. Definitely, the numerical data do not show a linear trend as would be expected if the experimental scaling law held. In Fig. 7, a log-log plot reveals instead that the spreading dynamics exhibits two distinct powerlaw regimes of the form:

$$
W(t)-W\left(t_{0}\right) \sim t^{m, n},
$$

where $m$ and $n$ are two distinct exponents relative to the two observed spreading behaviors.

The first regime (i.e., $\left.\left(t-t_{0}\right) / T \leq 16\right)$ is super-diffusive: the scaling $m$ is of order of 0.8 . The second regime is characterized by a scaling exponent $n<0.5$, thus corresponding to a slower dynamics and to a sub-diffusive spreading. The respective values of $m$ and $n$ obtained for the different acceleration rates $\Gamma$ are listed in Table II. We observe a slight variation of the scaling exponents with the acceleration rate: $m$ ranges from 0.61 to 0.81 , while $n$ takes values from 0.34 to 0.48 . There is no clear variation trends in $m$ and $n$ with increasing acceleration.

At this stage, it is important to emphasize that the second sub-diffusive regime found in the simulation is compatible with that observed in the experiments by Sanchez et al. [8]. We indeed obtain a scaling exponent close to $1 / 3$.

We analyze another important parameter, the solid volume fraction $\phi$, which may vary during the spreading due to the vibrations. Since this system is invariant

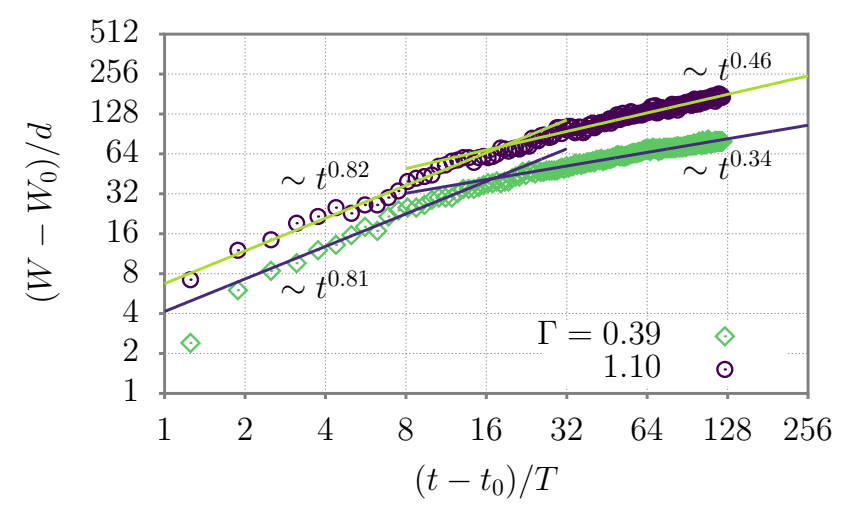

FIG. 7. A log-log plot of the granular droplet contact width on a rough substrate $(\lambda=1)$ for acceleration rates $\Gamma=0.39$ and 1.10. Two different spreading regimes are evidenced. 


\begin{tabular}{cccccccccc}
\hline \hline$\Gamma$ & 0.39 & 0.49 & 0.62 & 0.80 & 1.10 & 1.58 & 2.47 & 4.39 & 6.32 \\
\hline$m$ & 0.81 & 0.70 & 0.76 & 0.78 & 0.82 & 0.72 & 0.71 & 0.73 & 0.61 \\
\hline$n$ & 0.34 & 0.40 & 0.42 & 0.44 & 0.46 & 0.47 & 0.48 & 0.40 & 0.43 \\
\hline \hline
\end{tabular}

TABLE II. Critical exponents $m$ (super-diffusive) and $n$ (subdiffusive to diffusive) of the log-log spreading scaling laws for substrate bumpiness $\lambda=1$ at different acceleration rates $\Gamma$.

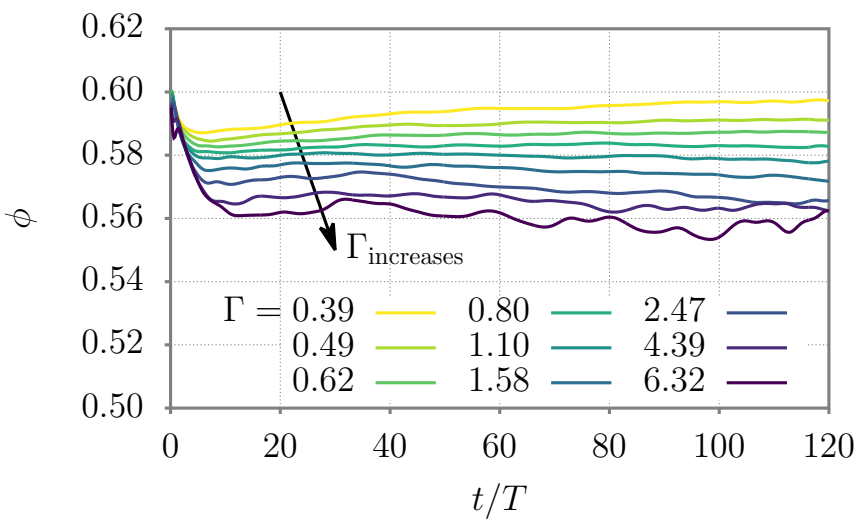

FIG. 8. Evolution of the mean packing fraction $\phi$ averaged over the granular droplet depth in the case of rough substrate $\lambda=1$ at different acceleration rates $\Gamma$.

along the $y$ direction, $\phi$ is computed as the ratio of two surfaces $\phi=S_{s} / S_{r}$, where $S_{s}$ is the intersection surface of a vertical $(x, z)$ plane at a given $y$ position with the three dimensional assembly of spheres, and $S_{r}$ is the reference surface area below the droplet profile. The value of the packing fraction $\phi$ is averaged through the droplet width along the $y$ direction. In Fig. 8, we plot the temporal evolution of the mean solid fraction of the granular droplet. In the transient stage (i.e., $t<5 T$ ), we observe a decompaction process which increases in intensity for increasing acceleration: the higher $\Gamma$, the more the decompaction is effective. After this transient, the packing fraction $\phi$ keeps roughly constant in course of time. We can however note a slight re-compaction of the granular droplet in the case of the smallest acceleration rates $(\Gamma=0.39$ and 0.49$)$.

\section{Bumpiness influence}

We now examine the influence of the basal bumpiness on the spreading dynamics. Fig. 9 presents the temporal evolution of the width $W$ and height $H$ of a granular droplet on four different substrates with respective bumpiness values $\lambda=0,0.25,0.5$ and 1 , obtained at a vibration acceleration $\Gamma=1.10$. As expected, the spreading rate increases with decreasing bumpiness values. The increase of the spreading rate is spectacular when we go from $\lambda=1$ to $\lambda=0.5$ but is much weaker for lower values of $\lambda$.

It is important to note that we do not observe signif-

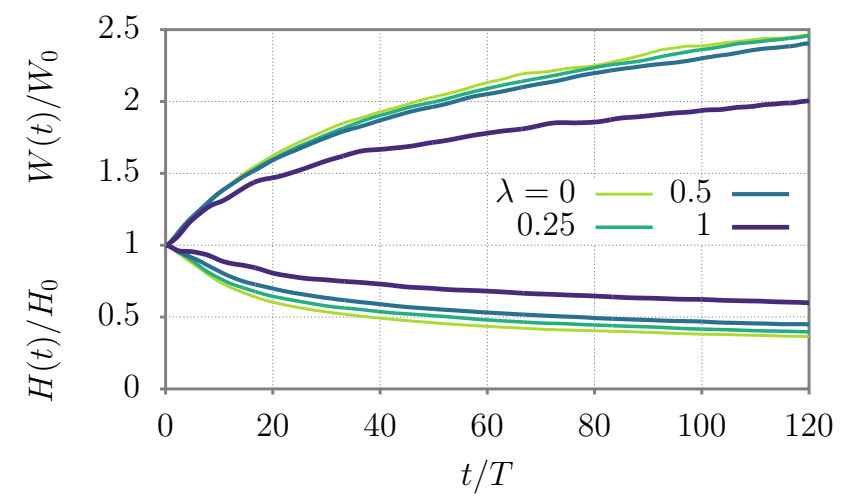

FIG. 9. Time evolution of $W$ relatively to its initial value $W_{0}$ for different substrate bumpiness $\lambda$ at the same acceleration rate $\Gamma=1.10$ (upper part). The height ratio $H / H_{0}$ evolution is also represented (lower part).

icant variation of the exponents $m$ and $n$ of the scaling laws with the substrate bumpiness (see Table III). We still have a first super-diffusive regime followed by a subdiffusive one.

\begin{tabular}{ccccc}
\hline \hline$\lambda$ & 0 & 0.25 & 0.5 & 1 \\
\hline$m$ & 0.81 & 0.85 & 0.78 & 0.82 \\
\hline$n$ & 0.47 & 0.50 & 0.49 & 0.46 \\
\hline \hline
\end{tabular}

TABLE III. Critical exponents $m$ (super-diffusive) and $n$ (sub-diffusive to diffusive) of the spreading scaling laws for different basal bumpiness $\lambda$ at the same acceleration rate $\Gamma=1.10$.

Additionally, we analyze the influence of the basal bumpiness on the temporal evolution of the solid volume fraction of the droplet during the spreading (see Fig. 10). Interestingly, lowering the bumpiness results in a greater re-compaction. For the smooth case $(\lambda=0)$ the packing fraction of the droplet at the end of the spreading process (i.e., $t / T=120)$ is $7 \%$ greater than that in the roughest case $(\lambda=1)$. The compaction phenomenon observed for low bumpiness is interpreted as an ordering process of the granular packing induced by the smooth base.

Finally, we shall say a few words about the combined effect of the bumpiness and the vibration acceleration. For finite bumpiness (i.e., $\lambda \neq 0$ ), the spreading rate is found to have a nonlinear dependence with the vibration period $T$ (see Fig. 5). In contrast, the smooth case is peculiar in the sense that the spreading becomes linearly dependent of the period $T$. As seen in Fig. 11, the curves $H(t / T)$ and $W(t / T)$ obtained for different accelerations surprisingly all collapse on each other. 


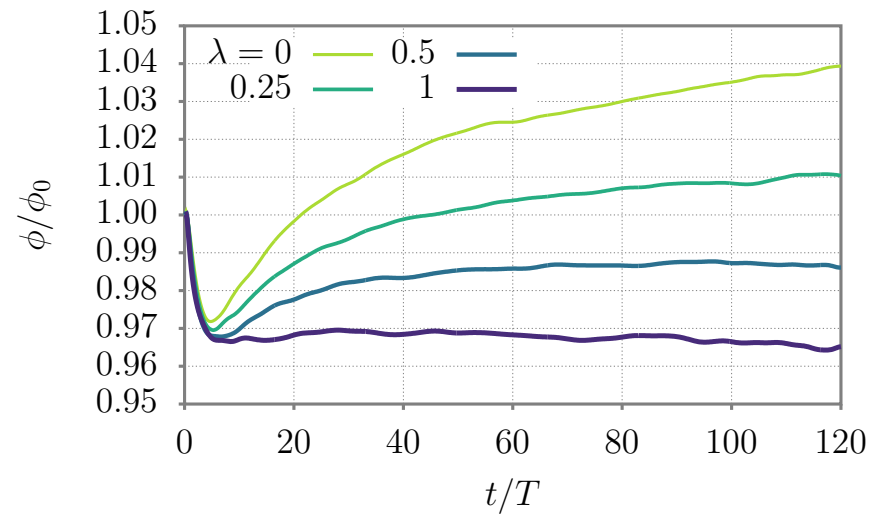

FIG. 10. Normalized solid packing fraction comparison for different substrate bumpiness at the same acceleration rate $\Gamma=1.10, \phi_{0}$ is the packing fraction before activating the vibrations.

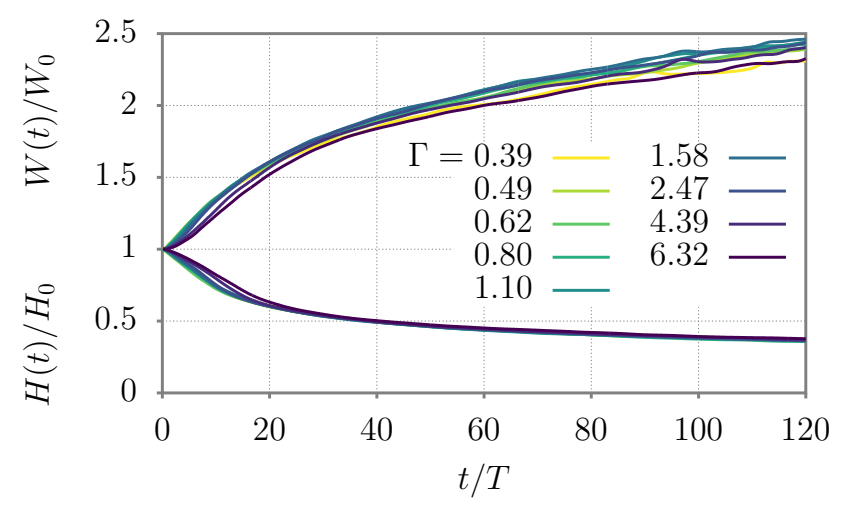

FIG. 11. Normalized width and height comparison for different accelerations at the same bumpiness $\lambda=0 . W_{0}$ and $H_{0}$ are, respectively, the initial width and height.

\section{Velocity field within the droplet}

We looked into the velocity field map obtained by a spatial averaging through the droplet width and a temporal one over a time span of two vibration periods. We observe a marked difference in the velocity field for the two spreading regime. In the first spreading regime $(t / T=5)$ (see the upper map in Fig. 12 and the vertical velocity profiles in Fig. 13). The flowing phase is localized close to the free surface: it spans from the top of the droplet down to the toe and penetrates the droplet interior over a thickness of about ten grain diameters. The spreading dynamics at this stage corresponds to the super-diffusive regime in which the flow appears to be controlled by superficial processes similar to avalanches on a granular pile. In contrast, the flow in the second sub-diffusive spreading regime (see Figs 12 and 13) is no longer localized at the free surface but spans more uniformly through the droplet depth. As a result, the spreading process is essential governed by a bulk flow spanning through the droplet depth rather than a surface flow.

\section{E. Basal friction}

The spreading process can be figured out in terms of momentum transfer between the granular droplet and the base. One way to understand how momentum is transferred from the base to the heap, the whole granular droplet can be seen, in a first approximation, as a solid mass sliding on a substrate with an effective friction coefficient $\mu$ which is defined as the ratio of the shear stress $\tau$ to the pressure $P$.

In Fig. 14, we present the time evolution of the effective friction. We observe a first phase during which $\mu$ increases monotonously. This increasing phase corresponds to the super-diffusive spreading regime.

In a second phase, $\mu$ goes towards a stabilization of the friction coefficient This second phase matches up with the sub-diffusive regime identified by a spreading scaling law approaching $t^{1 / 3}$ as reported in [8]. In this latter study, a nonlinear diffusion model was developed to explain the $1 / 3$ exponent. It is based on two assumptions: (i) a constant effective friction coefficient $\mu$ and (ii) a shallow flow approximation. The latter is justified as long as the vertical velocity are much smaller than their horizontal counterparts. These two assumptions are evidenced in our simulations during the sub-diffusive regime. While we have seen that the effective friction is indeed constant (see Fig. 14), the vertical profiles of the horizontal and vertical velocity in Fig. 13 indicate clearly that at $t=30 T$ the vertical velocities are much smaller than the horizontal ones: $v_{z} \sim-0.02 \sqrt{g d}$ and $v_{x} \simeq 0.1 \sqrt{g d}$.

The question that naturally arises is why the subdiffusive spreading regime is not observed in the initial phase of the spreading? In the first regime, the friction coefficient is not constant but most importantly the hypothesis of shallow flow does not hold anymore. Indeed, we clearly see on the velocity field map (see Fig. 12) that in the first regime, the flow is localized at the free surface, contrarily to the second regime in which the flow affects the whole granular droplet. In the first regime, the spreading is thus governed by a surface flow similar to avalanches on a granular pile. This surface flow is more efficient in spreading the droplet and leads to a spreading scaling law with a higher exponent. A more quantitative analysis work remains to be done to be able to derive the value of the exponent. This is what we aim to develop in a near future using a 2D continuum model with an appropriate rheological law.

\section{F. Effective rheology}

We look at the effective rheology in the second asymptotic sub-diffusive regime where $\mu$ is nearly constant during the spreading. The recent models concerning the rheology of granular matter [9, 19-23] show that dense unidirectional granular flows can be fairly well described using a single friction coefficient that varies with an inertial dimensionless parameter $I$, defined as the ratio of a 

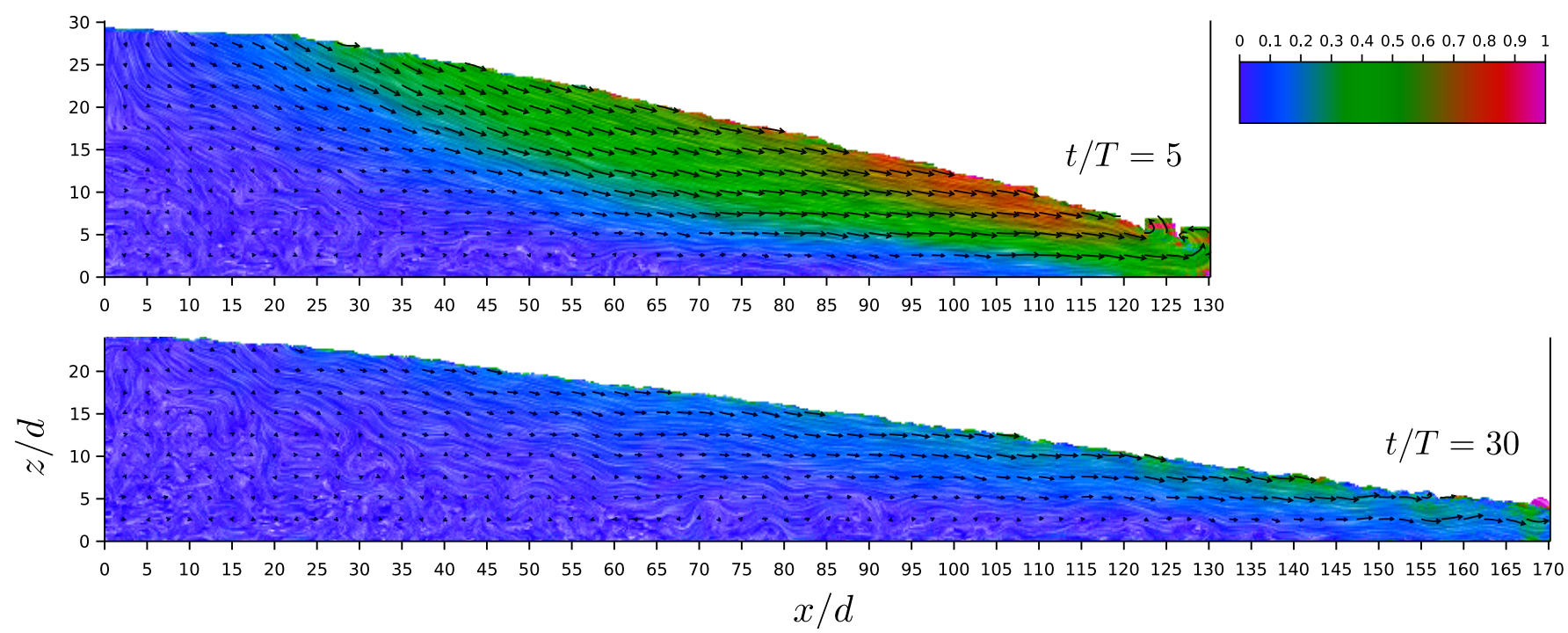

FIG. 12. Profiles of the coarse-grained vector and scalar velocity fields averaged over two vibration periods. All the velocities are normalized by the maximum velocity in both configuration $t / T=5$ (top) and at $t / T=30$ (bottom). The substrate bumpiness is $\lambda=1$ and acceleration $\Gamma=1.10$. We show only the half of the droplet since the spreading is symmetrical.
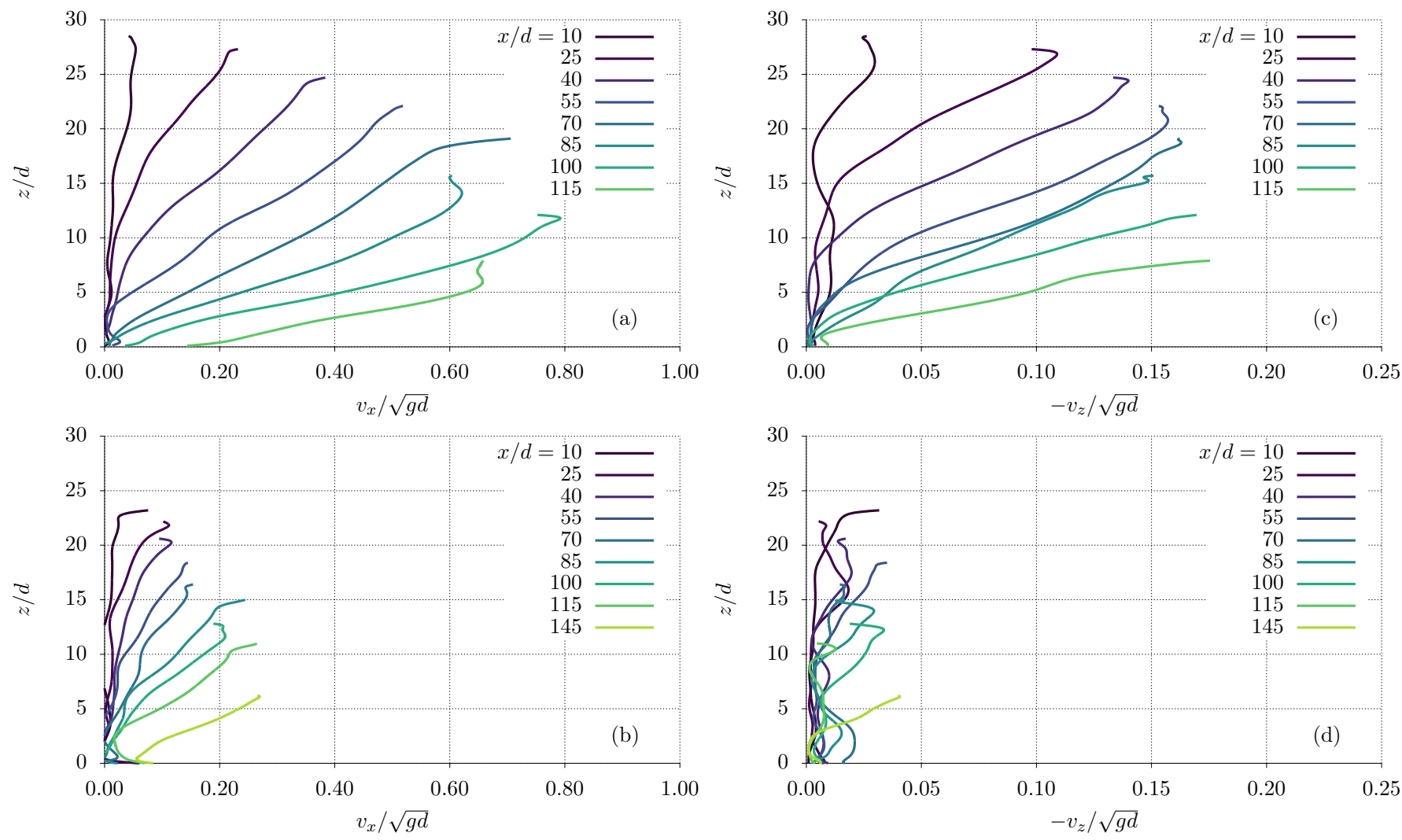

FIG. 13. Vertical profiles of the horizontal and vertical velocity $\left(v_{x}\right.$ and $\left.v_{z}\right)$ calculated at $t=5 T((\mathrm{a})$ and $(\mathrm{c}))$ and $t=30 T$ ((b) and (d)) respectively.

microscopic grain rearrangement time scale to a macroscopic flow time scale. This rheology which may be seen as a generalization of the basic Coulomb friction model, with a friction coefficient that varies according to the lo- cal shear rate $\tau$ and confinement pressure $P$, is usually written as follows:

$$
\mu(I)=\tau / P=\mu_{s}+\frac{\mu_{\infty}-\mu_{s}}{1+I_{0} / I}
$$




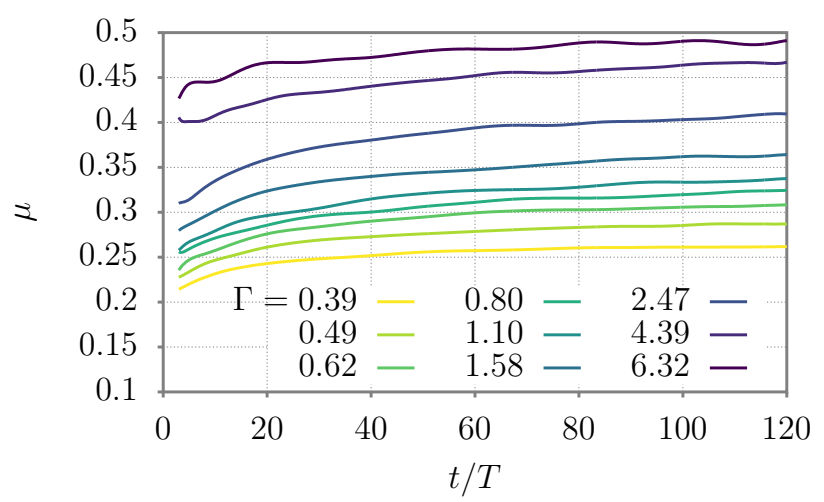

FIG. 14. Time evolution of the effective friction coefficient $\mu$ for different acceleration rates $\Gamma$ at constant bumpiness $\lambda=1$.

The constants $\mu_{s}$ and $\mu_{\infty}$ correspond, respectively, to a minimum friction coefficient at low $I$ and to an asymptotic friction coefficient at high $I$ values. In the case of the spreading of a granular droplet, the macroscopic time is associated with the duration for a grain to travel a distance $d$ under the shear velocity $a \omega$ while the microscopic time is given by the duration of a free grain to fall over a distance $d$ under gravity. This thus gives: $I=t_{\text {micro }} / t_{\text {macro }}=(d / \sqrt{g d}) /(d / a \omega)=a \omega / \sqrt{g d}$, which is simply the ratio of two velocity scales.

In our numerical study, this inertial number $I$ is varied from 0.6 to 2.5. The magnitude of the inertial number $I$ gives usually an indication about the nature of the granular flow [24]. In the limit of vanishing $I\left(I \leq 10^{-3}\right)$, the flow can be considered as shear independent ( $\mu$ is constant and equal to $\mu_{s}$ ). For intermediate values of $I$ (i.e., $10^{-2} \leq I \leq 10^{-1}$ ), this is the so-called dense inertial regime with a rate dependent flow. At higher inertial number $I \geq 1$, the flow gets faster and more dilute and quits the frictional regime governed by eq. 11 to enter a collisional regime which is well described by the kinetic theory for dissipative granular gas [25].

The frictional $\mu(I)$-rheology is usually complemented by an additional relationship which states that the solid fraction $\phi$ is a decreasing function of the sole parameter $I$. During the last decade, a large number of experimental and numerical studies confirms the relevance of the frictional $\mu(I)$-rheology for unidirectional dense granular flows as we report in Table IV.

In the present work, we analyze the asymptotic spreading regime in the framework of the frictional $\mu(I)$ rheology. As there is one-to-one correspondence between the inertial number and the reduced vibration acceleration, it is relatively easy to compute the variation of the effective friction coefficient $\mu$ of the granular droplet with the base and the mean solid volume fraction $\phi$ as a function of the inertial number. The computed values of $\mu$ and $\phi$ in our study are both averaged over two vibration periods during the second regime of the spreading.

In Figures 15 and 16, we present the resulting curves

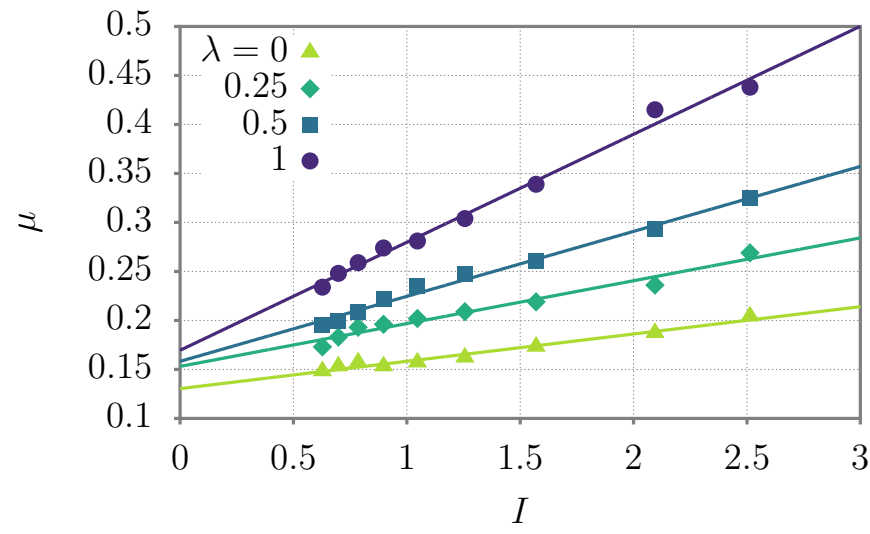

FIG. 15. Effective friction coefficient $\mu$ for different bumpiness values $\lambda$. Straight lines are the best fits.

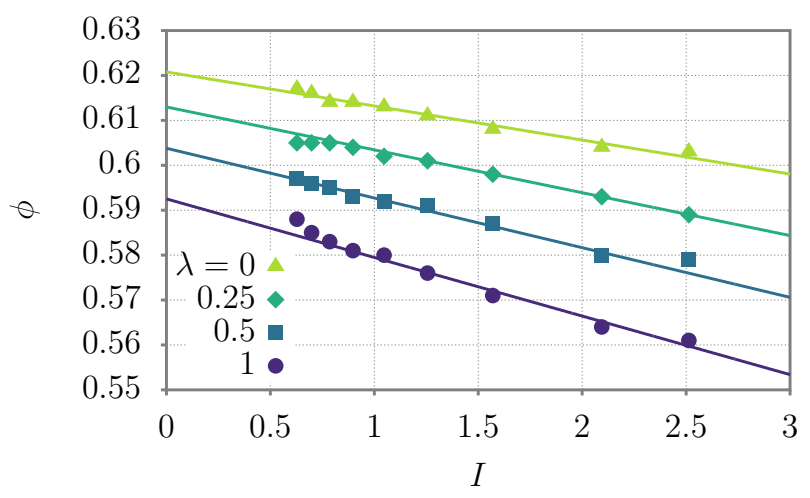

FIG. 16. Mean packing fraction for different bumpiness values $\lambda$. Straight lines are the best fits.

$\mu(I)$ and $\phi(I)$ obtained for the four different basal bumpiness. Concerning the effective friction, we can first note that the obtained values never exceeds the microscopic friction used in the simulation (i.e., $\mu<\mu_{p}=0.5$ ). Second, we observe a nice linear behavior up to high inertial numbers. The effective friction coefficient $\mu$ can therefore be well approximated by an affine function of $I$

$$
\mu(I)=\mu_{0}+a I .
$$

The fit coefficients $\mu_{0}$ and $a$ are given in Table V. $\mu_{0}$ shows a slight dependence with the base bumpiness: it increases from 0.13 to 0.17 when $\lambda$ goes from 0 to 1 . In contrast, the slope $a$ exhibits a strong dependence of the base bumpiness since it rises from 0.03 to 0.11 . Third, our results differ from those predicted by the model developed in [8], where the effective friction coefficient reveals a non-monotonous behavior. It presents a maximum seen as a transition from a shear thickening regime at low $I$ and a shear thinning at higher values.

The solid packing fraction $\phi$ also exhibits a linear variation with the inertial number $I$ that can be approximated by

$$
\phi(I)=\phi_{0}-b I
$$




\begin{tabular}{lllll}
\hline \hline Flow & Study & $I$ & $\mu(I)$ law & References \\
\hline Plane shear & 2D-DEM & $\leq 0.3$ & $\mu_{0}+a I$ & {$[19]$} \\
Plane shear & 2D-DEM & $\leq 0.1$ & $\mu_{0}+a I^{\alpha}$ & {$[26,27]$} \\
Plane shear & 3D-DEM & $\sim 1$ & $\mu_{0}+a I$ & {$[28]$} \\
Annular shear & 2D-DEM & $\leq 0.1$ & $\mu_{0}+a I+b e^{-(I / C)}$ & {$[29]$} \\
Annular plane shear & Experimental & $\leq 0.1$ & $\mu_{0}+a I$ & {$[30,31]$} \\
Pile between rough sidewalls & Experimental & $\leq 0.5$ & Eq. $(11)$ & {$[23]$} \\
Vibro-fluidized granular film & Experimental & $\sim 1$ & $a-b\left(I-I_{c}\right)^{2}$ & {$[8]$} \\
Vibro-fluidized and vane shear & Experimental & {$\left[10^{-5}, 10\right]$} & $\widetilde{\mu}(I)$ & {$[32]$} \\
\hline \hline
\end{tabular}

TABLE IV. Non-exhaustive summary of the $\mu(I)$ rheology laws found in the literature. The $\widetilde{\mu}(I)$ equation in [32] represents a unified rheological model based on a modified Pouliquen law in addition to other corrections due to Bernoulli pressure and Bagnold rheology.

\begin{tabular}{ccccc}
\hline \hline$\lambda$ & 0 & 0.25 & 0.5 & 1 \\
\hline$\mu_{0}, a$ & $0.13,0.03$ & $0.15,0.04$ & $0.16,0.07$ & $0.17,0.11$ \\
$\phi_{0}, b$ & $0.62,0.01$ & $0.61,0.01$ & $0.60,0.01$ & $0.59,0.01$ \\
\hline
\end{tabular}

TABLE V. Fitting parameters of the effective friction $\mu=$ $\mu_{0}+a I$ and the packing fraction $\phi=\phi_{0}-b I$ corresponding to different basal bumpiness $\lambda$.

The fit coefficients $\phi_{0}$ and $b$ are given in Table V. The dependence of these coefficients on the basal bumpiness contrasts with that obtained for $\mu_{0}$ and $a$. We find that the slope $b$ is weakly dependent on the basal bumpiness while $\phi_{0}$ decreases significantly with an increasing bumpiness parameter $\lambda$. It is noteworthy that the value of the slope $b$ of order of 0.01 is smaller than the standard value of 0.2 usually reported in the literature. We also note that the behavior of the effective friction coefficient $\mu$ and the packing fraction $\phi$ law is akin to those seen in the case of cohesive granular dense flows. The basal bumpiness $\lambda$ induces similar effects to those of the intensity of cohesion on the variations of $\mu(I)$ and $\phi(I)$ [33].

\section{CONCLUSION AND OUTLOOK}

Using discrete element method simulations, we studied the spreading of a granular droplet on a horizontally vibrated substrate. We used a variety of substrates with different bumpiness. We show that during a short transient, the granular droplet takes a parabolic shape as observed experimentally and then adopts a triangular shape for later times up to the end of the simulation. We also find out that the spreading dynamics is governed by two distinct regimes, a super-diffusive at the early stages, followed by an asymptotic sub-diffusive regime. These results contrast with the experiment where we only observe the second sub-diffusive regime [8]. We have identified that the super-diffusive regime is driven by surface flows similar to avalanches on a granular pile, while the subdiffusive regime is governed by a bulk flow that can be modeled by a nonlinear diffusion equation leading to a $1 / 3$ scaling exponent as shown in [8]. The surface flows are found to be much more efficient to spread the droplet and leads to a super-diffusive regime.

The transition is also marked by a change in the evolution of the basal friction. Indeed, in the first regime, the effective friction coefficient increases during the spreading while in the second regime, the friction reaches a plateau.

Varying the basal bumpiness has no strong impact on the droplet morphology nor on the scaling laws of the spreading: the triangular shape of the profiles is persistent and the spreading is still characterized by two distinct regimes with scaling exponents weakly dependent of the bumpiness.

Finally, we observe in the asymptotic sub-diffusive regime that the basal friction coefficient is a linear function of the inertial number. We also show that the packing fraction is a decreasing linear function of the inertial number in accordance with the frictional $\mu(I)$ rheology. Importantly, our simulation reveals that the effective friction coefficient and the droplet packing fraction are strongly dependent on the base bumpiness.

As a further work, it would be worthwhile to develop a quantitative analysis based on continuum model in order to derive the value of the exponent in the super-diffusive regime. The key issue is to use an appropriate rheological law. The first attempt would be the use of the classical $\mu(I)$-rheology or non-local rheological laws as such proposed in [26, 34].

\section{ACKNOWLEDGMENTS}

This work has been supported by the Algerian-French program CMEP-TASSILI No. 05MDU 644. This work was granted access to the HPC resources of UCI-UABT, Unité de Calcul Intensif of the University Aboubekr Belkaïd of Tlemcen, financed by the DGRSDT, Direction Générale de la Recherche Scientifique et du Développement Technologique. S.M.K. heartfully thanks B. Andreotti, P. Claudin and E. Clément from the 
Physique et Mécanique des Milieux Hétérogènes at ESPCI-Paris for the subject idea and related suggestions.
Special thanks to the staff of Physique des Milieux Divisés at Institut de physique, Université de Rennes 1, for support and thoughtful discussions.
[1] D. Bonn, J. Eggers, J. Indekeu, J. Meunier, and E. Rolley, Rev. Mod. Phys. 81, 739 (2009).

[2] A.-L. Biance, C. Clanet, and D. Quéré, Phys. Rev. E 69, 016301 (2004).

[3] P. Kavehpour, B. Ovryn, and G. H. McKinley, Colloids and Surfaces A: Physicochemical and Engineering Aspects 206, 409 (2002).

[4] D. R. Heine, G. S. Grest, and E. B. Webb, Phys. Rev. E 68, 061603 (2003).

[5] D. R. Heine, G. S. Grest, and E. B. Webb, Phys. Rev. E 70, 011606 (2004).

[6] D. R. Heine, G. S. Grest, and E. B. Webb, Langmuir 21, 7959 (2005), pMID: 16089405.

[7] Nadler, S., Bonnefoy, O., Chaix, J.-M., Thomas, G., and Gelet, J.-L., Eur. Phys. J. E 34, 66 (2011).

[8] I. Sánchez, F. Raynaud, J. Lanuza, B. Andreotti, E. Clément, and I. S. Aranson, Phys. Rev. E 76, 060301 (2007).

[9] GDR MiDi, Eur. Phys. J. E 14, 341 (2004).

[10] M. Allen and D. Tildesley, Computer Simulation of Liquids (Oxford: Clarendon Pr, 1987).

[11] J. Shäfer, S. Dippel, and D. E. Wolf, J. Phys. I France 6, 5 (1996).

[12] A. Lorenz, C. Tuozzolo, and M. Y. Louge, Experimental Mechanics 37, 292 (1997).

[13] L. E. Silbert, D. Ertaş, G. S. Grest, T. C. Halsey, D. Levine, and S. J. Plimpton, Phys. Rev. E 64, 051302 (2001).

[14] N. Brodu, P. Richard, and R. Delannay, Phys. Rev. E 87, 022202 (2013).

[15] L. E. Silbert, Phys. Rev. Lett. 94, 098002 (2005).

[16] S. Luding, International Journal of Solids and Structures 41, 5821 (2004), granular Mechanics.

[17] C. Salueña and T. Pöschel, Eur. Phys. J. E 1, 55 (2000).
[18] A. Stukowski, Modelling and Simulation in Materials Science and Engineering 18, 015012 (2010).

[19] F. da Cruz, S. Emam, M. Prochnow, J.-N. Roux, and F. m. c. Chevoir, Phys. Rev. E 72, 021309 (2005).

[20] O. Pouliquen and Y. Forterre, Journal of Fluid Mechanics 453, 133-151 (2002).

[21] P. Jop, Y. Forterre, and O. Pouliquen, Journal of Fluid Mechanics 541, 167-192 (2005).

[22] O. Pouliquen, C. Cassar, P. Jop, Y. Forterre, and M. Nicolas, Journal of Statistical Mechanics: Theory and Experiment 2006, P07020 (2006).

[23] P. Jop, Y. Forterre, and O. Pouliquen, Nature 441, 727 EP (2006).

[24] B. Andreotti, Y. Forterre, and O. Pouliquen, Granular Media: Between Fluid and Solid (Cambridge University Press, 2013).

[25] C. K. K. Lun, S. B. Savage, D. J. Jeffrey, and N. Chepurniy, Journal of Fluid Mechanics 140, 223-256 (1984).

[26] M. Bouzid, M. Trulsson, P. Claudin, E. Clément, and B. Andreotti, Phys. Rev. Lett. 111, 238301 (2013).

[27] A. Favier de Coulomb, M. Bouzid, P. Claudin, E. Clément, and B. Andreotti, Phys. Rev. Fluids 2, 102301 (2017).

[28] T. Hatano, Phys. Rev. E 75, 060301 (2007).

[29] G. Koval, J.-N. Roux, A. Corfdir, and F. m. c. Chevoir, Phys. Rev. E 79, 021306 (2009).

[30] A. Fall, G. Ovarlez, D. Hautemayou, C. Mézière, J.-N. Roux, and F. Chevoir, Journal of Rheology 59, 1065 (2015).

[31] A. Fall, M. Badetti, G. Ovarlez, F. Chevoir, and J.-N. Roux, EPJ Web Conf. 140, 03005 (2017).

[32] A. Gnoli, A. Lasanta, A. Sarracino, and A. Puglisi, Scientific Reports 6, 38604 EP (2016), article.

[33] P. G. Rognon, J.-N. Roux, M. Naaïm, and F. Chevoir, Journal of Fluid Mechanics 596, 21-47 (2008).

[34] K. Kamrin and G. Koval, Phys. Rev. Lett. 108, 178301 (2012). 